\title{
LA CIENCIA COGNITIVA Y EL ESTUDIO DE LA MENTE
}

\author{
Nicolas Medina C. ${ }^{1}$ \\ Universidad Nacional Mayor de San Marcos, Perú \\ (RECIBIDO EL 07/01/2008, ACEPTADO EL 30/05/2008)
}

\begin{abstract}
RESUMEN
En la actualidad se observa un desarrollo vigoroso de la ciencia cognitiva, que es un campo multidisciplinario en el que confluyen varias disciplinas, como la psicología cognitiva, la inteligencia artificial, la lingüística, la filosofía, la neurociencia, la antropología cognitiva, entre otras, con la finalidad de estudiar la cognición. El estudio de la cognición y la mente se ha venido realizando desde el punto de vista de la filosofía estrictamente especulativa, desde la perspectiva de la filosofía de la mente y desde el punto de vista científico. Los aportes hipotéticos y/o teóricos de esos estudios de la mente son discutidos y, en la mayoría de los casos, sometidos a un análisis experimental para contrastar su validez científica. En ese sentido, la ciencia cognitiva ha ido estableciendo las propiedades esenciales de la mente y la cognición, tal como el carácter representacional de la mente, la relación causal entre la representación cognitiva y la conducta, el carácter simbólico de la información representada mentalmente, la mente como procesador de la información, la mente como un sistema de mecanismos, la modularidad de la mente, etc. Sin embargo, en la ciencia cognitiva actual es notoria la presencia de diferentes enfoques cognitivos que defienden el modelo simbólico clásico, por un lado, y por otro están aquellos que defienden el modelo conexionista o procesamiento distribuido en paralelo (PDP), y también aquellos que postulan modelos mentales no representacionalistas. En conclusión, se puede decir que hoy en día el estudio de la mente y la cognición es una empresa importante en el que están comprometidas varias disciplinas.
\end{abstract}

Palabras clave: Ciencia cognitiva, Mente, Procesamiento de información, Representación mental, Arquitectura cognitiva, Modelo simbólico clásico, Modelo conexionista, Modelos no representacionalistas.

\begin{abstract}
At present we observe a vigorous developmental of the cognitive science like a multidisciplinary field that involve several disciplines, such as cognitive psychology, artificial intelligence, linguistics, philosophy, neuroscience, and cognitive anthropology, in order to study the cognition. The study of cognition and mind has been made from a point of view of the speculative philosophy, or from perspective of the philosophy of mind, or from a point of view scientific. The hypothetical and/or theoretical proposals offered from those studies, are discussed and are submitted to experimental analysis, for the purpose to test their scientific

1 Docente Principal de la Facultad de Psicología de la Universidad Nacional Mayor de San Marcos, Lima- Perú. E-mail: medina0712@yahoo.com
\end{abstract}


validity. Thus, the cognitive science set the essential properties of mind and cognition, such as the representational nature of mind, the causal relation between the cognitive representation and the behavior, the symbolic nature of information mentally represented the mind like processor of information, the mind like a system of mechanisms, the modularity of mind, etc. But, at the actual cognitive science is relevant the presence of different cognitive viewpoints, on the one hand, are those that defend the classic symbolic model, on the other hand, are those that sustain the connectionist model or parallel distributed processing (PDP), and others that support mental models non representational. In conclusion, the actual study of mind and cognition is an important enterprise that involves several disciplines.

Keywords: Cognitive science, Mind, Information processing, Mental representation, Cognitive structure, Classic symbolic model, Connectionist model, Mental models non representational.

\section{INTRODUCCIÓN}

El interés por la comprensión de la naturaleza y funcionamiento de la mente humana comenzó hace más de dos mil años. Los filósofos y pensadores de aquella época estudiaron el problema de la cognición humana desde una perspectiva predominantemente especulativa. Sólo hace poco más de cien años (con la creación del laboratorio de psicología experimental en Leipzig, 1879), la mente es estudiada desde un punto de vista científico, y a partir de la década de 1960 el enfoque cognitivo surgió como un paradigma importante, proponiendo alternativas convincentes frente a las insuficiencias teóricas y epistemológicas del conductismo. En ese sentido, muchos investigadores de esa época se interesaron por el estudio de la mente, concordando con la idea que el paradigma cognitivo posee el marco teórico y metodológico más adecuado para formular y estudiar el problema de la naturaleza de la cognición humana.

La definición del modelo cognitivo fue una contribución de la ciencia cognitiva, que es conocido como un movimiento interdisciplinar que nació como producto del Simposio sobre Teoría de la Información, realizado en el año de 1956 por el Instituto de Tecnología de Massachusetts (Massachusetts Institute of Technology-MIT). En ese simposio se reunieron investigadores de diversas disciplinas con el propósito de intercambiar ideas sobre comunicación y ciencias humanas. Según Medler (1998), en esa reunión destacaron tres conferencias que dieron origen al movimiento de la ciencia cognitiva, que fueron: The magical number seven de G. Miller, Three models of language de N. Chomsky y The logic theory machine de A. Newell y H. Simon.

Basada en esas conferencias, comenzó a surgir la idea que consideró a las contribuciones de la psicología experimental humana, la lingüística teórica y la simulación de los procesos cognitivos mediante la computadora, como elementos constituyentes de un gran conjunto. De esa manera, se formó la noción que para comprender el funcionamiento de la cognición humana era preciso combinar los esfuerzos de diferentes disciplinas. En consecuencia, actualmente la ciencia cognitiva se define como el estudio interdisciplinar de la mente, en la que participan las siguientes disciplinas: Ciencia de la computación e Inteligencia artificial, Lingüística, Neurociencia, Filosofía y Psicología, entre otras. Aunque cada una de esas disciplinas tenga su propia interpretación de la ciencia cognitiva, ellas están articuladas dentro de un conjunto coherente, a través del principio central que declara que la 
mente es un procesador de la información, eso quiere decir que la mente recibe, conserva, recupera, transforma y transmite información. Esa información y su procesamiento pueden ser analizadas como patrones cognitivos, que a su vez pueden ser objeto de manipulación. Además, se supone que esos procesos son estados representacionales y semánticos que se realizan dentro de las restricciones físicas del cerebro.

Sin embargo, en la actualidad no todos los científicos cognitivos concuerdan con esa concepción de la mente, sino, por el contrario, existen diversos puntos de vista sobre la teoría y la metodología para estudiar la mente y su relación funcional con el lenguaje. En ese sentido, se puede ver el destaque de los puntos de vista que sostienen el carácter representacionalista de la mente (e.g., el modelo cognitivo simbólico clásico y el modelo conexionista), los que caracterizan la mente como no representacionalista (e.g., el realismo incorporado, según Johnson y Lakoff) y los enfoques dinámicos y emergentistas de la mente.

\section{ENFOQUES DE ESTUDIO DE LA MENTE}

Desde una perspectiva general, el estudio de la mente puede ser realizado desde los puntos de vista de la filosofía especulativa, la filosofía de la mente y la ciencia de la mente. Respecto al estudio predominantemente filosófico, tradicionalmente ha girado alrededor de especulaciones sobre problemas relacionados con la caracterización ontológica de las representaciones mentales o con la naturaleza de la intencionalidad, dando como resultado diversos sentidos al concepto de "mente". De esa manera, algunas veces "mente" fue empleado en sentido de "intelecto", particularmente en el significado de "intelecto pasivo"; otras veces "mente" significaba "espíritu"; también significó "psique" u "operaciones psíquicas en general". En algunas ocasiones se ha preferido el concepto "mente" en vez de "espíritu", para evitar las implicaciones metafísicas que envuelve el último término. También, frecuentemente se ha entendido por "mente" el entendimiento, particularmente el entendimiento después de haber entendido o comprendido algo. Asimismo, se ha analizado la "mente" relacionándola significativamente con el alma como agente intelectual que usa la inteligencia, adoptando el concepto "mente" un sentido primariamente "intelectual" (ver Ferrater Mora, 1979, 1986; p. 2182). Cabe señalar que esos diferentes sentidos del concepto "mente" fueron utilizados para caracterizar la entidad abstracta e ideal de la mente como distinto de "cuerpo", que constituye una entidad física, material. Esa diferenciación subyace en el clásico problema de la relación mente-cuerpo, hasta hoy en debate.

Desde la perspectiva de la filosofía de la mente, el estudio de la mente realiza especulaciones sobre la base de los resultados de trabajos científicos multidisciplinares. De modo que, desde esa perspectiva de la filosofía de la mente toda aportación científica fue bien recibida; esas aportaciones han provenido de la lingüística, la psicología, la inteligencia artificial, las ciencias cognitivas, la robótica, la biología evolucionista, la antropología, la etología, las neurociencias, la neuropsicología, la neurofisiología, la neurocomputación, la lógica y la teoría de la computabilidad, las matemáticas de los sistemas dinámicos, la filosofía del lenguaje. De esa manera, el carácter especulativo de la filosofía de la mente asumió una actitud más objetiva. Al respecto, Broncano (1995) afirmó que "la naturaleza de la mente ha dejado de ser el territorio exclusivo de los psicólogos; es un territorio abierto a la especulación" (p. 12). 
En la actualidad, la filosofía de la mente ha derivado en dos vertientes de discusión sobre la mente, una es la psicología popular (folk psychology) o de sentido común, en el que destacan como representantes Fodor y Dretske, y otra es el eliminativismo, que tiene como representantes a Churchland y Stich. En relación a la psicología popular plantea explicaciones y predicciones de la conducta apelando a estados mentales con contenido sobre creencias, deseos, miedos, intenciones, intuiciones y, en general, estados psicológicos construidos en términos proposicionales, considerados como causas de la conducta. Por ejemplo, es una explicación de la psicología popular el hecho de que Pedro haya ido a la fiesta porque deseaba ver a Isabel y creyó que ella se encontraría allí. También es una predicción típica de la psicología popular cuando alguien predice que María abrirá la refrigeradora sobre la base de su deseo de comer un pedazo de torta y su creencia de que la torta se encuentra en la refrigeradora. Esas son explicaciones obvias, de sentido común, para las cuales no se necesita ser un profesional en psicología para explicarlas.

Respecto al eliminativismo o materialismo eliminativista, es la posición contraria a la psicología popular, porque considera que las creencias y deseos son términos vacíos de contenido significativo, o sea que no tienen correspondencia con ninguna entidad física. Así, el eliminativismo sostiene que nada que no sea físico puede actuar como causa de las conductas o acciones; de modo que la apelación que realiza la psicología popular a estados intencionales como responsables causales de la conducta es una apelación improcedente. En consecuencia, el eliminativismo sostiene que las categorías mentales implicadas en la explicaciones del sentido común (psicología popular) son términos vacíos, i.e., no refieren a ninguna realidad física, y que la adscripción de estados mentales caracterizados intencionalmente es, por tanto, un procedimiento errado para explicar la conducta.

En cuanto se refiere al enfoque científico de la mente, el estudio de la mente se realiza en el contexto de la observación y la experimentación, construyendo hipótesis o conjeturas acerca de la estructura y funcionamiento de las actividades mentales, que son contrastadas empíricamente con las manifestaciones físicas de la mente, o sea, con las conductas. En esta tarea científica los estudiosos de la mente han recurrido a los expedientes conceptuales de la epistemología moderna, con la finalidad de analizar la mente con criterios objetivos, construyendo modelos y/o utilizando metáforas como estrategias heurísticas que posibilitan la construcción de teorías científicas. En ese sentido, el aporte epistemológico más importante estaría dado por la reconceptualización de la naturaleza de los hechos y el carácter hipotético-deductivo de las teorías científicas.

Sobre la reconceptualización de la naturaleza de los hechos, se puede decir que en la construcción y desarrollo de la ciencia no es exclusiva la concepción inductivista, en el que los hechos constituyen la materia prima para elaborar teorías, sino también juega un papel importante la concepción teórica del científico para determinar la naturaleza de los hechos, es decir, los hechos pueden considerarse como una construcción del científico (e. g., cuando se construyen modelos en el dominio de la ciencia cognitiva). Y respecto a la naturaleza hipotético-deductiva de la teoría, se considera que la justificación del carácter hipotético-deductivo de las teorías científicas no sólo se realiza por la fuerza de los datos fácticos, sino también por las concepciones de principio (en el que intervienen significativamente las metáforas), e inclusive por las formulaciones causales de tipo teleológico. Esa reconceptualización epistemológica, superior al positivismo lógico, ha influido en el 
desarrollo del cognitivismo y en la construcción teórica de la psicología cognitiva y ramas afines, que cada vez está tornándose más constructivista que empirista.

Actualmente, un grupo significativo de científicos cognitivos comparten el punto de vista que considera la mente humana como un complejo sistema que recibe, almacena, recupera, transforma y transmite información. Esas operaciones realizadas sobre la información son denominadas computaciones o procesos de información, motivo por el cual la mente es concebida como un sistema computacional o de procesamiento de información. Stillings et al. (1995) señalaron algunos conceptos fundamentales relacionados con la concepción computacional de la mente, que implica procesamiento de la información, que serán descritos brevemente:

a) Los procesos de información permiten al organismo (o sistema) realizar respuestas sistemáticas frente a alguna variedad de condiciones del ambiente. Esas respuestas son típicamente adaptativas o dirigidas a metas; de modo que los procesos de información tienen la cualidad de ser significativos en relación al entorno, es decir, la información en el organismo (o sistema) es algo relacionado con el mundo, como teniendo contenido, significado o sentido. Tales cualidades de contenido a veces son denominadas cualidades semánticas o intencionales. Esas cualidades permiten atribuir entendimiento, creencia o conocimiento para cualquier organismo (o sistema) que actúa adaptativamente en respuesta a la información tomada del medio ambiente. La comprensión del proceso de información por el organismo (o sistema) permitirá explicar el contenido de la información que trata, la que al mismo tiempo explicará su competencia para emplear esa información al servicio de determinadas metas.

b) Los procesos de información tienen carácter representacional, o sea, la información que figura en un sistema computacional debe ser representada en alguna forma. Un buen entendimiento de la operación de computación demanda una comprensión de cómo la información está representada en la mente. Esa representación se traduce en símbolos, por ejemplo, el "5" es un símbolo que representa al 5, que es el número que está siendo representado.

c) Los procesos de información pueden ser descritos formalmente, que implica una relación con la noción de algoritmo, que es definido en términos de procesos que operan sobre la representación. Cabe aclarar que los procesos algorítmicos no operan sobre el dominio que está siendo representado, ni son definidos en términos del significado de la representación, lo cual es realizado independientemente por la función semántica, desde la representación al dominio que está siendo representado. Un algoritmo es un procedimiento o sistema formal, que es definido más en términos de la forma de la representación que su significado. Es un asunto puramente de manipulación de patrones en la representación. Por ejemplo, el algoritmo para la multiplicación manual de número decimales (de 0 a 9) es completamente una cuestión de manipulación de listas de símbolos de dígitos de acuerdo con ciertas reglas de operación para multiplicar.

d) Los conceptos arriba mencionados son empleados por los científicos cognitivos para alcanzar un básico conocimiento científico de los procesos de información. Es decir, los científicos cognitivos procuran descubrir los principios de explicación general y fundamental del procesamiento de información. 


\section{CIENCIA COGNITIVA}

La ciencia cognitiva es un campo interdisciplinario, de base empírica, preocupado por el estudio de la naturaleza de la mente humana. Según Gardner, en ese estudio también están comprometidos aspectos epistemológicos, motivo por el cual dicho autor definió la ciencia cognitiva "como un empeño contemporáneo de base empírica para responder a interrogantes epistemológicos de antigua data, en particular los vinculados a la naturaleza del conocimiento, sus elementos componentes, sus fuentes, evolución y difusión" (Gardner, 1985, 1987; p. 21). En virtud de ello, se puede decir que la ciencia cognitiva es una nueva forma de abordar el antiguo problema de la naturaleza del conocimiento humano, por medio de la observación, la experimentación y la medición.

Stillings et al. (1995) refieren que el origen histórico de la ciencia cognitiva fue motivado por el interés que tuvieron los filósofos de la antigua Grecia en el estudio del razonamiento deductivo, que es el proceso por el cual uno supone alguna información como verdadera y a partir de ella deriva lógicamente más información. Por ejemplo, desde las premisas Todos los perros tienen pulgas y Duke es un perro, uno puede deducir lógicamente la conclusión Duke tiene pulgas, que constituiría una nueva información. En ese sentido, la teoría del razonamiento silogístico de Aristóteles explicó que los argumentos deductivamente válidos frecuentemente son extraídos de una pequeña cantidad de enunciados generales. Entonces, se puede decir que el aprendizaje para razonar deductivamente puede ser concebido como la adquisición de la habilidad de procesar información, por medio del cual formas válidas de argumentos pueden ser reconocidos y producidos. En el siglo XVII, los filósofos Leibniz y Hobbes sustentaron que el pensamiento humano realiza cálculos (conjeturas) con información no numérica. Hoy los lingüistas y filósofos continúan estudiando las propiedades lógicas del lenguaje, mientras que los psicólogos cognitivos comparan los procesos de razonamiento actual de la gente con los sistemas idealizados creados por los filósofos y matemáticos, y los investigadores en inteligencia artificial diseñan programas de computadoras que simulan el razonamiento lógico.

La ciencia cognitiva concibe que el conocimiento humano es un sistema que siempre está recogiendo, almacenando, recuperando, transformando, transmitiendo y actuando sobre la información. De modo que se supone que el ser humano debe poseer conocimiento para actuar. En relación a la naturaleza del conocimiento, actualmente es motivo de discusión entre los científicos cognitivos, pero en la mayoría hay ciertas coincidencias para considerar un carácter representacional del conocimiento. En esa perspectiva, se puede decir que el ser humano, como ser cognoscente, actúa sobre la base de sus representaciones. La experiencia de un organismo en relación a determinados acontecimientos cargados de información, dan como resultado la formación de una representación cognitiva, por medio de la cual se facilita el uso de reglas para las representaciones, que permitirían inferir ciertas creencias sobre el mundo, o sea, diseñar inferencias y construir hipótesis plausibles.

En ese sentido, de acuerdo con los fines de la ciencia cognitiva, la representación cognitiva humana es descrita en función de símbolos, esquemas, imágenes, ideas y otras formas de representación mental. Basado en esas representaciones el sujeto cognoscente actúa. Al respecto, Pylyshyn $(1984,1989)$ sostuvo que conociendo las representaciones que poseen los sujetos cognoscentes, y considerando el supuesto de que sus conductas están conectadas 
con sus representaciones mediante ciertos principios generales, es posible explicar un importante segmento de las regularidades conductuales exhibidos por dichos sujetos. Sin embargo, aquí se levanta el siguiente problema: ¿Cómo es posible que un sistema físico (asumiendo que los sujetos cognoscentes son sistemas físicos) actúe sobre la base de un "conocimiento de" objetos y relaciones con los que el sistema no está causalmente conectado de manera apropiada? Es cierto que los objetos relativos a los temores y deseos de los sujetos no causan la conducta de la misma forma en que las fuerzas y energías causan conductas en el dominio físico.

Con la finalidad de explicar la naturaleza de la relación causal entre la representación cognitiva del sujeto y su conducta, es pertinente referir un ejemplo que da Pylyshyn (1984, 1989) al respecto. Así, cuando un sujeto tiene el deseo de conseguir la olla conteniendo oro, que se encuentra en el final del arco iris, determina causalmente la conducta de ir en busca de dicha olla. Es obvio que la olla de oro (que no existe) no es una causa semejante a la que está implicada en una ley natural. En el caso del ejemplo, el responsable de la conducta de ir en busca de la olla de oro es una creencia o "representación", cuyo contenido semántico es la meta esperada.

Cabe observar que en la actualidad, es público el debate entre los científicos cognitivistas sobre la forma más conveniente de conceptualizar la naturaleza de las representaciones mentales. Así, algunos consideran que hay una sola forma de representación mental realizada a través de proposiciones o enunciados; otros opinan que hay por lo menos dos formas de representación mental, una más parecida a figuras o imágenes y otra más próxima a las proposiciones; hay otro grupo que postula múltiples formas de representación mental.

Además de la discusión sobre el carácter representacional del conocimiento, la ciencia cognitiva considera otro aspecto significativo que también es motivo de debate, en este caso se trata del asunto relativo al supuesto de que el conocimiento humano actúa como un procesador de información, lo cual conduce a la concepción de la computadora como modelo del pensamiento. Esa concepción está estrechamente ligada con el carácter representacional de la mente, en el sentido de que lo que hace posible que los sujetos actúen sobre la base de representaciones es que ellos establecen representaciones en forma física, por medio de códigos cognitivos, y que su conducta es una consecuencia causal de operaciones realizadas sobre esos códigos. En vista de que eso es precisamente lo que hacen las computadoras, entonces, se puede decir que la cognición es un tipo de computación (Pylyshyn, 1984, 1989). Siendo así, un programa de computadora que multiplica números o administra una base de datos, obviamente está ejecutando operaciones formales sobre la información (traducida en códigos cognitivos), que tienen una relación representacional directa de un dominio particular. El éxito de esos programas se debe enteramente al hecho de que las mencionadas operaciones formales sobre los códigos cognitivos preservan la relación representacional.

Por su parte, Gardner $(1985,1987)$ señaló que no todos los científicos cognitivos concuerdan con el modelo de la mente como una computadora; sin embargo, la mayoría ha sentido la influencia de dicho modelo. Así, el modelo de la computadora ha sido útil en dos aspectos del estudio de la mente: por un lado, se afirma que la computadora actúa como una prueba 
de existencia de la mente, eso quiere decir que si una máquina construida por el hombre razona, tiene metas, revisa y corrige su conducta, transforma información, etc., entonces los seres humanos merecen ser caracterizados con esas mismas propiedades. Por otro lado, además de servir de modelo del pensamiento, la computadora también es una herramienta importante en el trabajo de los científicos cognitivistas, de modo que la mayoría utiliza la computadora para analizar sus datos, y otro grupo de cognitivistas cada vez mayor procura simular los procesos cognitivos computacionalmente. Al respecto, Gardner realizó el siguiente comentario referente a las posibles consecuencias producidas por el desarrollo de la ciencia de la computación, señalando que "la ciencia de la inteligencia artificial, elaborada en torno de la simulación por computadoras, es considerada por muchos la disciplina central de la ciencia cognitiva y la que más probabilidades tiene de desplazar o volver superfluos otros campos de estudio" (Gardner, 1985, 1987; p. 57).

\section{CONCEPTO DE MENTE}

De acuerdo con el punto de vista de la ciencia cognitiva, la mente humana es un sistema complejo que recibe, almacena, recupera, transforma y transmite información. Esas operaciones sobre la información son llamadas computaciones o procesos de información, que delinean la concepción de la mente desde una perspectiva computacional o de procesamiento de la información. Esa orientación se basa en lo que más hace la gente, que siempre está percibiendo, almacenando, recuperando, transformando, transmitiendo y actuando sobre la base de un conjunto de información. Obviamente, esa información proviene del mundo exterior. En ese sentido, se puede decir que existe un proceso de transacción entre el organismo y el medio ambiente externo, en el que el organismo juega un papel importante, ya que constituye el elemento fundamental que ejecuta el procesamiento de la información proveniente del medio externo. Al respecto, Newell $(1990,1994)$ sostuvo que la mente es el sistema de control que guía el comportamiento del organismo en su compleja interacción con el mundo dinámico real. En otras palabras, se puede decir que mente es el nombre adoptado para referirse al sistema de control desarrollado dentro del organismo, con la finalidad de realizar las interacciones adecuadas en beneficio del organismo o, por último, para la sobrevivencia de la especie.

En suma, desde el punto de vista de la psicología cognitiva simbólica, se puede decir que la mente se caracteriza por ser representacional, basada en símbolos, sobre los cuales es posible operar formalmente, lo que también proporciona el carácter computacional de la mente. Cabe señalar que esos símbolos son portadores últimos de las propiedades semánticas y de la intencionalidad, y que al mismo tiempo pueden poseer significado y ser objetos físicos. En ese sentido, la mente se concibe como una máquina simbólica, basada en la conexión establecida entre las nociones de símbolos e intencionalidad. La capacidad intencional de la conducta de un organismo se supone que se origina en el interior del propio organismo, i. e., la conducta inteligente de algunos organismos está causada por determinados estados del propio organismo, llamados estados mentales. Esos estados mentales se caracterizan por poseer un contenido intencional representado internamente, y constituyen una representación cognitiva que puede ser una creencia, deseo, temor, recuerdo, etc. Esa concepción de los estados mentales implicó la noción de "actitudes 
proposicionales" propuesto por Jerry Fodor, que funciona como causa de las conductas y poseen significado (ver Fodor, 1987, 1994).

\section{ARQUITECTURA DE LA MENTE}

Un aspecto importante en la ciencia de la cognición es la teoría de la arquitectura cognitiva, por medio de la cual se describe el marco estructural en el que se realiza el procesamiento de la información, y consecuentemente sería responsable de las restricciones fundamentales de la conducta del organismo (o sistema). Específicamente, la arquitectura cognitiva se refiere al diseño y organización de la mente, que determina que el sistema cognitivo posea la característica de tener una estructura y una función. En ese sentido, se puede decir que un sistema cognitivo que posea una determinada estructura, produce una conducta que depende de la realización de una función en dicha estructura. Al respecto, Ezquerro (1995) caracterizó la arquitectura cognitiva de la siguiente manera:

"[...] El término arquitectura se usa para indicar que la estructura de un sistema de procesamiento de información posee un carácter permanente u originario, con lo que habría que entender por arquitectura, en términos muy generales, la estructura fija que proporciona el marco dentro del cual tiene lugar el procesamiento cognitivo. Desde esta perspectiva, se podría afirmar que, en sentido abstracto, los componentes de la arquitectura reflejan o representan las estructuras físicas subyacentes del sistema". (Ezquerro, 1995; p. 100)

Por su parte, Stillings et al. (1995) señalaron que los psicólogos cognitivos han tratado de configurar cuál es el diseño total, o arquitectura, de la mente humana, con la finalidad de averiguar qué tipo de capacidades generales de procesamiento de información tendría una mente, para hacer las múltiple actividades cognitivas que realiza. Al mismo tiempo se aclara que la arquitectura cognitiva no determina el alto grado de flexibilidad de la cognición humana, del pensamiento y de las conductas específicas, sino que dicha arquitectura es considerada como un conjunto abstracto de mecanismos que potencializan una gran variedad de capacidades. En ese sentido, es importante encontrar la estructura subyacente de la conducta humana, que implicaría descubrir la arquitectura mental, que sería el substrato de las capacidades básicas del procesamiento de información, y que en última instancia permitiría adaptar adecuadamente las respuestas del organismo frente a una amplia variedad de situaciones (Stillings et al., 1995; p. 16).

Desde el punto de vista computacional, Fodor (1984) consideró importante el establecimiento de la arquitectura cognitiva, que permitiría explicar su funcionamiento, ya sea en términos físicos o biológicos, o simplemente podría ser caracterizada en términos funcionales mientras los mecanismos biológicos no sean conocidos (Fodor, 1984; Preface, p. xvi). Por otro lado, con la finalidad de distinguir "arquitectura" y "proceso cognitivo", cabe mencionar la explicación que Pylyshyn (1984) hace al respecto, señalando lo siguiente: existe una distinción entre regularidades de la conducta que pueden ser causadas o por las propiedades físicas o biológicas del sistema orgánico, o por sus representaciones (como creencias y metas). Paralelamente, esta distinción se relaciona con aquella que hay entre los procesos gobernados por principios semánticos (llamados "procesos cognitivos") y aquellos gobernados por la denominada "arquitectura funcional" del sistema. Esta última es un término prestado de la ciencia de la computación, en la que el término "arquitectura 
funcional" se usa para referir al conjunto de recursos básicos del sistema computacional, que puede ser el "hardware" o el "software", disponibles para la creación de programas (Pylyshyn, 1984; p. 130).

Actualmente, desde la perspectiva cognitiva del procesamiento de información, el estudio de la arquitectura de la mente se viene realizando de acuerdo con dos modelos paradigmáticamente diferentes, conocidos como el modelo clásico simbólico y el modelo conexionista (o procesamiento distribuido en paralelo-PDP), ambos compartiendo la posición de ser representacionalistas.

\section{MODELOS DE ARQUITECTURAS COGNITIVAS}

\section{Modelo simbólico clásico}

Tradicionalmente, el punto de vista del procesamiento de información ha sido caracterizado de acuerdo con la hipótesis del sistema de símbolo físico de Newell y Simon, que constituye el fundamento teórico del modelo simbólico clásico de la ciencia cognitiva. Básicamente, esa hipótesis declara que la cognición se fundamenta en patrones de información que pueden ser representados en forma de símbolos, y esos símbolos pueden ser manipulados. Es por eso que a veces se ha considerado que la arquitectura de la mente es la arquitectura de las computadoras de von Neumann. Desde esa perspectiva, se afirma que el lenguaje es un sistema que usa alguna señal física (un sonido, un gesto, una marca en el papel) para expresar un significado, y que los sistemas de procesamiento de información son mecanismos que representan la información en forma simbólica, que son computacionales, o sea, esas representaciones simbólicas pueden ser manipuladas y transformadas para crear nuevas representaciones (Stillings et al., 1995).

En ese sentido, en la concepción clásica simbólica de la arquitectura de la mente es central la idea de representación proposicional, que sostiene que las proposiciones son unidades completas del pensamiento que pueden tener un valor de verdad, i. e., pueden ser verdaderas o falsas. De modo que cualquier unidad lingüística simple es incompleta, por ejemplo, María no es una proposición completa, ni ama a es una proposición completa; en cambio, María ama a Juan sí es una proposición completa, porque expresa una idea completa que puede ser verdadera o falsa. Eso significa que las proposiciones son unidades funcionales importantes en el procesamiento cognitivo y tienen un considerable poder para representar el mundo, lo cual está relacionado con el aspecto semántico de las oraciones representadas en la mente representacional.

\section{Modelo conexionista o procesamiento distribuido en paralelo (PDP)}

En la década de 1980 varios investigadores desarrollaron una concepción alternativa frente al enfoque clásico simbólico, adoptando diferentes denominaciones, como: conexionismo, procesamiento distribuido en paralelo, redes neurales artificiales y paradigma subsimbólico. La denominación genérica que involucra a esas variantes alternativas es el término conexionista, de modo que esa nueva alternativa de la arquitectura cognitiva se la conoce como el modelo conexionista. Este modelo conexionista se basa en el procesamiento paralelo 
de subsímbolos, utilizando propiedades estadísticas en vez de reglas para transformar información; mientras que el modelo clásico simbólico usa reglas explícitas, frecuentemente lógicas, organizadas jerárquicamente para manipular símbolos en forma serial.

Además, el modelo conexionista se fundamenta en las contribuciones de la neurofisiología del cerebro, para explicar la naturaleza de la cognición. Basado en esa concepción, el conexionista considera que la unidad básica del cerebro es la neurona, que tiene seis propiedades funcionales básicas, que son: es un mecanismo de input que recibe señales del medio ambiente o de otras neuronas; es un mecanismo que conduce la información integrada en intervalos de distancia; es un mecanismo de output que envía información a otras neuronas o células; es un mecanismo computacional que mapea un tipo de información dentro de otro; es un mecanismo representacional que promueve la formación de representaciones internas. Esas características sirvieron para modelar las redes neuronales, que vendrían a ser la base del procesamiento distribuido en paralelo (PDP).

Medler (1998) señaló que Rumelhart, Hinton y McClelland (1986) registraron ocho propiedades esenciales de los modelos de procesamiento distribuido en paralelo (PDP), que son los siguientes: un grupo de unidades de procesamiento; un estado de activación; una función de output para cada unidad; un patrón de conectividad entre las unidades; una regla de propagación para los patrones de transmisión de actividades a través de la red de conexiones; una regla de activación para la combinación de las influencias de los input sobre una unidad con el estado actual de esa unidad, para producir un nuevo nivel de activación en la misma unidad; una regla de aprendizaje mediante la cual los patrones de conexiones son modificados por la experiencia; un medio ambiente dentro del cual el sistema debe operar.

Seguidamente, el mismo autor (Medler, 1998) refirió que Bechtel y Abrahamsen (1991) redujeron la lista anterior de propiedades a cuatro propiedades que caracterizan a los diferentes tipos de arquitectura conexionista, que son las siguientes: la conectividad de las unidades, la función de activación de las unidades, la naturaleza del proceso de aprendizaje que modifican las conexiones entre las unidades, y cómo la red es interpretada semánticamente.

En suma, las propiedades mencionadas del modelo conexionista pueden ser sintetizadas en tres principios fundamentales: 1) las señales, o símbolos, son procesadas por unidades elementales, 2) las unidades de procesamiento son conectadas en paralelo a otras unidades de procesamiento, y 3) las conexiones entre las unidades de procesamiento son ponderadas. Desde esa perspectiva, el procesamiento de las señales comprendería la recepción, transformación y transmisión de información. Las señales también pueden ser realizadas por medios eléctricos, químicos o mecánicos. Asimismo, las señales podrían ser establecidas por un estímulo externo (tal como la activación de la luz sobre la retina) o por otras unidades de procesamiento. Esas unidades de procesamiento pueden aludir a neuronas, o a funciones matemáticas o a los demonios de Selfridge. De modo que, basado en esos principios se puede observar un gran espectro de modelos (e. g., el Pandemonium de Selfridge, el Modelo de adquisición del tiempo pasado de Rumelhart y McClelland, el Modelo de correspondencia del movimiento de Dawson) que caen dentro de la clasificación de los estudios conexionistas. 
Los conexionistas asumen que las conexiones entre las unidades pueden ser, o no, masivamente paralelas, en el sentido que cada unidad es conectada a otra unidad. Esas conexiones pueden ser del tipo "feed-forward", o sea, señales que siguen una sola dirección hacia adelante, o pueden ser del tipo interactivo (feed-back), o sea, señales bidireccionales. Los conexionistas también consideran que los pesos asociados con las conexiones pueden ser: o "hard-wired", o aprendidos, o ambos. Cabe anotar que los pesos representan la fuerza de la conexión entre dos unidades; dicha fuerza puede ser excitatoria o inhibitoria.

\section{Modelo no-representacionalista según el realismo incorporado de Johnson y Lakoff}

Johnson y Lakoff (2002) asumieron que el "realismo incorporado" (embodied realism), originalmente denominado "experiencialismo", representa una alternativa frente a las concepciones filosóficas tradicionales del racionalismo y del empirismo. El realismo incorporado afirma que el lugar exacto de la experiencia, del significado y del pensamiento, es una serie continua de interacciones entre el organismo y el medio ambiente, que da como resultado nuestra comprensión del mundo. De acuerdo con esa concepción, no existe una separación entre la mente y el cuerpo, y siempre estamos "en contacto" con el mundo que nos rodea, mediante nuestros actos incorporados y experiencias.

Los mismos autores (Johnson y Lakoff, 2002) también señalaron que el experiencialismo no concuerda con la teoría de la representación, afirmando que la única teoría funcional de la representación es aquella que concibe la representación como un patrón flexible de interacciones entre el organismo y el medio ambiente, y no es una entidad mental interna que, de algún modo, está enlazada con las partes del mundo externo mediante una extraña relación de "referencia". En ese sentido, el realismo incorporado rechaza la noción clásica de "representación", junto con las ideas de significado y referencia, que son los elementos que sustentan la teoría de la representación.

En consecuencia, desde el punto de vista del realismo incorporado, representación es un término inadecuado para explicar la característica de la cognición humana, en la medida que ese término designa un modelo cognitivo idealizado de la mente, que concibe ideas de objetos internos no incorporizados, que de alguna forma corresponden a estados que ocurren en el mundo externo. Sosteniendo en cambio, que ni los sistemas ideales ni cualquier otro aspecto de la estructura conceptual son "representaciones". O sea, esa concepción experiencialista, o realismo incorporado, considera que un esquema ideal es una estructura neural relacionada con el sistema sensorio-motor, mediante el cual se establece el sentido de la experiencia. Al respecto, Johnson y Lakoff (2002) relataron que Rohrer (2001) demostró experimentalmente que la parte motora de la corteza cerebral (que está conectada funcionalmente con las actividades de las manos), no sólo es activo para la experiencia motora de la mano, sino también se relaciona funcionalmente con la experiencia lexical de las frases referentes a las manos, y con oraciones metafóricas en las que se utilizan las manos, como una fuente de dominio. De ahí se derivó el tipo de "estudio cerebral realista”, que constituye el sustento de la teoría del significado incorporado. 


\section{Modelo no-representacionalista según la cognición incorporada}

Este enfoque cognitivo sigue la misma línea conceptual que el anterior, y considera que el cuerpo juega un papel central en la formación de la mente. Los defensores de la cognición incorporada no adoptan una concepción de la mente trabajando sobre problemas abstractos, sino conciben al cuerpo como una entidad que demanda una mente para hacerlo funcionar. Al respecto Clark (1998) declaró que "los cerebros biológicos son los primeros y principales sistemas de control para los cuerpos biológicos. Los cuerpos biológicos se mueven y actúan en un rico mundo real circundante" (p. 506).

En las décadas tempranas de la psicología cognitiva, los sistemas perceptual y motor fueron considerados como simples mecanismos periféricos de input y output, y no como mecanismos para la comprensión "central" de los procesos cognitivos. Algunas ramas de las ciencias cognitivas han resaltado las funciones sensoriales y motoras, así como también su importancia para la exitosa interacción con el medio ambiente.

El punto de vista de la cognición incorporada postula la idea que la mente debe ser comprendida en el contexto de su relación con el cuerpo físico, que a su vez interactúa con el mundo. También sostiene que los seres humanos hemos evolucionado de criaturas cuyos recursos neurales primariamente se encargaron del procesamiento perceptual y motor, y su actividad cognitiva principal consistió en la interacción inmediata on-line con el medio ambiente. Por eso, la cognición humana antes que ser centralizada, abstracta y definidamente distinta de los módulos periféricos de input y output, tienen profundas raíces en el procesamiento sensorio-motor.

\section{CONSIDERACIONES FINALES}

En la actualidad, la ciencia cognitiva continúa con la antigua tarea (de la filosofía de la mente) de explicar, desde el punto de vista científico, la naturaleza de los fenómenos psicológicos y de la mente humana, y establecer las propiedades esenciales de las capacidades y procesos cognitivos. Para el cumplimiento de esa empresa, la ciencia cognitiva ha convocado la participación de varias disciplinas, que están tratando desde sus propias perspectivas los diversos aspectos implicados en la dinámica del conocimiento humano.

Cabe mencionar que el estudio científico de la mente humana es un proceso complejo, en el que la filosofía de la mente ha tomado una participación activa seria y relevante, proponiendo teorías, cuyos contenidos se expanden entre las diversas disciplinas comprometidas con el estudio de la mente, estimulando el debate. En esa perspectiva, terminaré este artículo describiendo someramente la teoría de los mecanismos subyacentes de la conducta (ver William Bechtell, 2005) y la teoría de la modularidad de la mente (ver Jerry Fodor, 1983).

\section{Sobre los mecanismos subyacentes en la conducta}

La ciencia cognitiva, así como la ciencia de la conducta, pretenden explicar la conducta de acuerdo con sus correspondientes puntos de vista. Así, la psicología conductual 
describe la conducta apelando a leyes que explican el establecimiento de vínculos causales entre los estímulos del entorno externo y el organismo comportamental. Mientras que la ciencia cognitiva recurre a los mecanismos cognitivos responsables de la producción de conductas.

Bechtel (2005) conceptualizó el mecanismo como un sistema organizado de partes componentes que realizan determinadas operaciones o funciones. Los componentes del mecanismo y su organización producen una conducta (p. 314). De acuerdo con ese concepto, un mecanismo es un sistema que opera en la naturaleza, y su explicación mecanicista es un producto epistémico. De modo que cuando los científicos cognitivos llegan a una explicación mecanicista, deben representar (a veces verbalmente, pero con frecuencia en forma de diagramas) las partes componentes y sus operaciones y la forma en que ellos están organizados.

La característica central de tales explicaciones mecanicistas es que descompone un sistema que produce conductas, en sus partes y operaciones componentes. Las partes y operaciones en que un mecanismo se descompone están estrechamente relacionadas: las partes relevantes son aquellas que ejecutan operaciones y por eso son partes operativas (working parts). En el sistema de un mecanismo se distinguen, por un lado, las partes comprendidas estructuralmente y, por otro lado, las operaciones comprendidas funcionalmente, de modo que la comprensión de un mecanismo requiere de ambos aspectos: estructural y funcional.

En relación a la naturaleza psicológica de las operaciones, Bechtel (2005) afirmó que las operaciones implicadas en los mecanismos responsables de las conductas, usan conceptos apropiados para describir las conductas en la que están comprometidos agentes cognitivos. Esta perspectiva es manifiesta en el enfoque cognitivo de manipulación simbólica, que constituye un modelo de actividad cognitiva. En este modelo, las operaciones psicológicas son conceptualizadas como transformaciones de las estructuras de los símbolos, donde la estructura de esos símbolos son construidos en forma de oraciones o frases en un lenguaje natural o formal. Basado en esas operaciones psicológicas implicadas en el mecanismo mental, Fodor propuso su hipótesis del "lenguaje del pensamiento" (language of thought), que sería la causa de las expresiones lingüísticas (Fodor, 1975).

Esas operaciones son parecidas a las que realizan los seres humanos cuando hacen tareas de escribir palabras y frases, digitándolas o en manuscrito, leyéndolas hacia atrás, o realizando otras alteraciones, etc. Pero la diferencia está en que esos símbolos son considerados para ser codificados de alguna forma dentro del cerebro de la persona, y las operaciones de lectura y escritura son operaciones internas, y no operaciones sobre el papel.

\section{Sobre la modularidad de la mente}

El concepto de modularidad ha sido ampliamente influyente en la ciencia cognitiva. La concepción de modularidad fue expuesta de manera sistemática en el libro de J. Fodor (1983), The modularity of mind (La modularidad de la mente). En ese libro, Fodor propuso una teoría general de la percepción y la cognición, basada en un sistema de mecanismos modulares de input y sistemas centrales de regulación del comportamiento. 
La propuesta substancial que hace Fodor es referente a su hipótesis sobre la constitución de dos sistemas en el dominio mental, que actúan paralelamente: un sistema representado por los mecanismos modulares de input, de carácter sensorio-perceptual, que sigue un recorrido bottom-up (de abajo para arriba), y otro sistema no modular representado por los sistemas centrales, que siguen una ruta top-down (de arriba para abajo).

El sistema modular de inputs tiene obligatoriamente las siguientes características:

a) Es un mecanismo de dominio específico.

b) La operación de input es obligatorio.

c) El acceso al nivel superior está limitado por los mecanismos de input.

d) El mecanismo de input es rápido.

e) El mecanismo de input es encapsulado.

f) Los analizadores de input realizan producciones poco elaboradas.

g) Los mecanismos de input están asociados con una arquitectura neural fija.

En relación a los sistemas centrales no modulares, que representan el nivel superior del sistema mental, cumplen la función de fijar las creencias por inferencia no demostrativa. Es decir, el sistema central se encarga de procesar la información entregada por los mecanismos de input y la información obtenida de la memoria, para decidir la elección de la "mejor hipótesis" sobre cómo debe ser el mundo.

\section{REFERENCIAS BIBLIOGRÁFICAS}

1. Bechtel, W. (2005). "The Challenge of Characterizing Operations in the Mechanisms Underlying Behavior". Journal of the Experimental Analysis of Behavior 84 (3): 313-325.

2. Broncano, F. (1995). La mente humana. Madrid: Editorial Trotta.

3. Clark, A. (1998). Embodied, situated, and distributed cognition. En W. Bechtel and G. Graham (Eds.) A companion to cognitive science. Malden, MA: Blackwell Publishers, pp. 506-517.

4. Ezquerro, J. (1995). "Teorías de la arquitectura de lo mental". En Fernando Broncano (ed.) La mente humana. Madrid: Editorial Trotta.

5. Ferrater Mora, J. (1979, 1986). Diccionario de Filosofía. Madrid: Alianza Editorial.

6. Fodor, J. A. (1983). The modularity of mind. Mass.: The MIT Press.

7. . (1975). The language of thought. Mass.: Harvard University Press.

8. . (1987, 1994). Psicosemántica: El problema del significado en la filosofía de la mente. Madrid: Editorial Tecnos. 
9. Gardner, H. (1985, 1987). La nueva ciencia de la mente: Historia de la revolución cognitiva. Buenos Aires: Editorial Paidós.

10. Johnson, M. \& Lakoff, G. (2002). "Why cognitive linguistics requires embodied realism". Cognitive Linguistics, 13 (3): 245-263.

11. Medler, D. A. (1998). “A brief history of connectionism”. Neural Computing Surveys, $1(2): 18-72$.

12. Newell, A. (1990, 1994). Unified theories of cognition. Mass.: Harvard University Press.

13. Pylyshyn, Z. W. (1984, 1989). Computation and Cognition: Toward a Foundation for Cognitive Science. Mass.: MIT Press.

14. Stillings, N. A., Weisler, S. E., Chase, C. H., Feinstein, M. H., Gardfield, J. L. \& Rissland, E. L. (1995). Cognitive Science: An Introduction. Mass.: The MIT Press.

15. Wilson, M. (2002). "Six views of embodied cognition". Psychonomic Bulletin \& Review, 9 (4): 625-636. 